\title{
BOUNDED RATIONALITY AND INCOMPLETE CONTRACTS ${ }^{*}$
}

\author{
by \\ Luca Anderlini \\ Southampton and Georgetown Universities \\ and \\ Leonardo Felli \\ London School of Economics and Political Science
}

\section{Contents:}

Abstract

1. Introduction

2. The Contracting Model

3. A Definition of Contractual Incompleteness

4. Computable Contracts

5. The Approximation Result

6. Complexity Costs

7. Some Related Results

8. The Structure of the State Space

Appendix and the Approximation Result

References

Discussion Paper

No. TE/00/407

December 2000
The Suntory Centre

Suntory and Toyota International Centres

for Economics and Related Disciplines

London School of Economics and Political

Science

Houghton Street

London WC2A $2 \mathrm{AE}$

Tel.: 020-7405 7686

\footnotetext{
A talk based on this paper was delivered as an invited lecture at the GAMES 2000 conference in Bilbao. We thank the participants, Nabil A-Najjar and Hamid Sabourian for very helpful discussions and comments. Part of the research work for this paper was done while Leonardo Felli was visiting the Department of Economics of the University of Pennsylvania. He wishes to thank them for their generous hospitality. Both authors gratefully acknowledge the ESRC for financial support (Grant R000237825).
} 


\begin{abstract}
This paper explores the link between boundedly rational behaviour and incomplete contracts. The bounded rationality of the agents in our world is embodied in a constraint that the contracts they write must be algorithmic in nature.
\end{abstract}

We start with a definition of contract incompleteness that seems both appealing and widely applicable. Our first task is then to show that, by itself, the algorithmic nature of contracts is not enough to generate genuinely incomplete contracts in equilibrium. As in Anderlini and Felli (1994), we call this the Approximation Result.

We then consider contractual situations in which the complexity costs of a contract are explicitly taken into accoaunt. We consider a broad (axiomatically defined) class of complexity measures and in this framework we show that incomplete contracts obtain in equilibrium.

We also extensively discuss some recent literature directly related to the results reported here.

Keywords: Incomplete contracts; bounded rationality; complexity costs.

JEL Nos.: C69, D81, D89

(C) Luca Anderlini and Leonardo Felli. All rights reserved. Short sections of text, not to exceed two paragraphas, may be quoted without explicit permission provided that full credit, including (c) notice, is given to the source. 


\section{Bounded Rationality and Incomplete Contracts}

\section{INTRODUCTION}

\subsection{Motivation}

Commenting on the state of play in the research agenda on incomplete contracting Tirole (1999, p. 773) writes: "Complexity matters because contracts are played by real players, who must not be daunted by hard-to-grasp equilibrium strategies".

This paper explores the link between boundedly rational behavior and the incompleteness of contracts.

Our analysis is largely based on the framework used in Anderlini and Felli (1994) and Anderlini and Felli (1999). We use this framework to highlight some of the difficulties that arise in generating contractual incompleteness from bounded rationality. We also discuss how this framework, once complexity costs are explicitly taken into account, can indeed generate endogenously incomplete contracts.

Once the framework is set up, our first task is to define formally what an incomplete contract is. This is not an uncontroversial issue. In a variety of different frameworks, many implicit or explicit ways of defining contract incompleteness have been put forth in the literature. These have been so varied that according to Tirole (1999, p. 743) again: "[...] there is unfortunately no clear definition of 'incomplete contracting' in the literature. While one recognizes one when one sees it, incomplete contracts are not members of a well-circumscribed family [...]". Of course, from a formal point of view, the definition we propose here applies to the model that we analyze and does not directly translate into a universally applicable one. However, we believe that it can be usefully extended to fit a wide variety of models (for instance to models with relationship-specific investments and/or asymmetric information, which we do not consider in this paper).

Our definition of an incomplete contract is based on a benchmark contract (which coincides with the first best in our symmetric information model), and on a test to be applied to any contract $x$. The result of the test, splits the set of all possible contracts into two exhaustive disjoint sets: complete and incomplete contracts. An intuitive description of what the test is meant to capture is as follows. Start by looking at the partition of the state space that the contract $x$ induces. Comparing this partition with the one induced by the benchmark contract, can we conclude that the parties who 
wrote contract $x$ were somehow 'constrained' in their ability to distinguish between states of nature? If the answer is 'yes' we call contract $x$ incomplete.

As in Anderlini and Felli (1994) our point of departure is to assume that contracts are algorithmic maps (Turing machines) between the state space and the actions to be taken by the two parties if a given state occurs (say the value of a sharing rule for the surplus generated by the trade). In this way, we model a 'limit case' of bounded rationality. Anything that can be computed by any imaginable finite device in a finite number of steps is algorithmic in the sense that we use here. This is a limit case in the sense that carrying out extra steps in a computation has no cost, but the number of steps must nevertheless be finite.

In a model with a countable state space (there is a continuum of states in Anderlini and Felli (1994)) we recover a version of a proposition that we refer to as the Approximation Result. On the one hand it is true that in some cases the first best sharing rule cannot be embodied in an algorithmic contract. On the other hand, given any contracting problem and any arbitrarily small number $\varepsilon$, there exists an algorithmic contract that guarantees that the parties' expected utilities are within $\varepsilon$ of their first best levels.

The Approximation Result can be viewed as a negative result in establishing the link between bounded rationality and contract incompleteness. Effectively, it seems to tell us that, at least in the limit case, bounded rationality in the formulation of the contract alone cannot generate any meaningful form of contractual incompleteness.

After having looked at a model that captures a limit case of bounded rationality as described above, we move on to a world in which complexity costs are explicitly taken into account (Anderlini and Felli 1999). The approach we take is to model complexity in an axiomatic way (Blum 1967), so that our definition of complexity encompasses a very wide variety of possible specifications of the complexity costs of a contract.

We consider a model in which the choice of contract is determined by the solution to a maximization problem, but the complexity costs of the contract itself are explicitly accounted for. In this set-up we find that endogenous contract incompleteness obtains: for any specification of the complexity costs, there exist contracting problems for which the complexity costs force the parties to write a contract that is 
incomplete in the sense described above.

After relating this work to some recent literature, we conclude by describing a result that may take us away from the Approximation Result mentioned above. Work by Al-Najjar, Anderlini, and Felli (2000) shows that if we allow for contracts that are contingent on some 'complex' events, then the restriction to algorithmic contracts alone may be sufficient to generate endogenously incomplete contracts that keep the parties' expected utilities bounded away from their first best values.

\subsection{Related Literature}

Starting from the seminal paper by Grossman and Hart (1986) a large and growing literature has analyzed the inefficiencies that arise in a world in which contracts are incomplete. These papers model a number of institutions, such as vertical and lateral integration (Grossman and Hart 1986), the allocation of ownership over physical assets (Hart and Moore 1990), the allocation of authority (Aghion and Tirole 1997) and power (Rajan and Zingales 1998) in organizations whose role is to reduce the inefficiencies induced by contractual incompleteness. The question that is not fully answered by this literature is why contracts are incomplete.

A more recent strand of literature has addressed this question and proposed the indescribability of key aspects of the contract as the reason why the resulting equilibrium contract may be incomplete. For example, the parties involved in a contractual relationship may have common knowledge of the states of nature in which they are required to undertake different performances but they may lack the ability, the language or the motivation to describe ex-ante these states in their contractual arrangement.

In particular if the authority enforcing the contract (the court) cannot verify ex-post whether a given state of nature has occurred, describing such a state in the ex-ante contract is of no use to the contracting parties (Hart and Moore 1988). Alternatively - even if the court can observes the realized state - the contracting parties may lack the necessary degree of rationality (the ability, the time, the language or the computational resources) necessary to describe exactly the various states of nature in the ex-ante contract they draw up (Anderlini and Felli 1994, Anderlini and Felli 1999, Krasa and Williams 1999, Battigalli and Maggi 2000, Al-Najjar, Anderlini, and Felli 2000). The latter is the explanation for contractual incompleteness that we 
discuss at length in this paper.

A recent paper by Maskin and Tirole (1999) raises a general objection to the attempt of explaining the incompleteness of contracts from the indescribability of states of nature. Maskin and Tirole (1999) argue that indescribability of the states of natures is not enough to prevent the parties from writing a complete contract. In short, they argue that the parties could write an ex-ante contract that commits them to play an ex-post revelation game. In this revelation game the parties are required to report the payoff relevant information associated to the realized states or any uniquely defined coding of this information. Provided that these payoffs (or their codes) are common knowledge and the game can be designed so that the parties in equilibrium report the truth, the allocation implemented by such a mechanism coincides with the allocation implemented by the best contract in the absence of any indescribability.

This critique, in principle, applies to our analysis as well. We have, however, two responses to Maskin and Tirole (1999). First, in the simple co-insurance model we analyze it is enough to assume that the parties' utilities exhibit constant absolute risk aversion to make sure that it is not possible to construct an ex-post revelation game that induces the parties to report the truth. ${ }^{1}$ Of course this assumption greatly limits the applicability of our approach.

Second, devising and describing the necessary coding of the indescribable states used for the revelation game á la Maskin and Tirole may be even more complex than devising and describing the first best contract. Hence, the same argument that shows that in some instances the first best contract is prohibitively costly would show that a message-contingent mechanism is also prohibitively costly.

The impact of the critique in Maskin and Tirole (1999) is also diminished by the possibility of re-negotiation. Two recent papers (Segal 1999, Hart and Moore 1999) show that if the parties to a contract are allowed to write message contingent mechanisms but they cannot commit ex-ante not to renegotiate the agreed mechanism if an ex-post mutually beneficial opportunity arises, the gain from these mechanisms may be greatly reduced as the complexity of the environment increases. ${ }^{2}$

\footnotetext{
${ }^{1}$ This statement is true if we restrict attention to full or unique implementation.

${ }^{2}$ For a closely related result see Reiche (1999).
} 


\subsection{Overview}

We start with the description of the basic contracting model in Section 2. In Section 3 we proceed to formalize the definition of contractual incompleteness that we sketched out above. Section 4 introduces the notion of an algorithmic or computable contract with which we work in the sequel. In Section 5 we show that a restriction to algorithmic contracts alone is not sufficient to generate contractual incompleteness. In Section 6 we introduce the notion of axiomatic complexity costs that are associated with a contract. In this section, we also show that for every complexity cost function satisfying the basic axioms there exist contracting environments such that the optimal contract given the complexity costs is in fact incomplete. In Section 7 we discuss at some length the related papers of Krasa and Williams (1999) and Battigalli and Maggi (2000). Finally, Section 8 is devoted to a discussion of the results in Al-Najjar, Anderlini, and Felli (2000).

For ease of exposition, all proofs are relegated to the Appendix.

\section{The Contracting Model}

The contracting problem that we consider is extremely simple. Two risk-averse agents face a risk-sharing problem. For simplicity, we work with a standard co-insurance model to be specified shortly. All the results in this paper generalize to a set-up like the one used in Anderlini and Felli (1994).

The randomness of the environment is entirely described by the possible realizations of a state of nature $s$. The set of possible states of nature is denoted by $\mathcal{S}$. Unless we note otherwise, $\mathcal{S}$ is assumed to be the set of natural numbers $\mathbb{N}$. It is important to notice at this point that all the results in Sections 3, 4, 5 and 6 below apply (in some cases trivially) to the case of finitely many states. The probability that state $s$ occurs is denoted by $p(s)$ throughout the paper.

The two agents in the model are indexed by $i=1,2$. Each agent's preferences are represented by a strictly increasing and concave Von-Neumann Morgenstern utility function for 'money' $V_{i}: \mathbb{R}_{+} \rightarrow \mathbb{R}$. For each $i=1,2$, we take $V_{i}$ to be bounded from below and to satisfy $V_{i}^{\prime}(0)=\infty$ and $V_{i}^{\prime}(\infty)=0$.

To each state of nature there corresponds a 'default allocation' denoted by the pair of non-negative reals $\left(d_{1}(s), d_{2}(s)\right)=d(s)$. These can be thought of as the 
endowments of the two agents in state $s$. The total of resources available in state $s$ is denoted by $r(s)$ so that $r(s)=d_{1}(s)+d_{2}(s)$. A contract is a function $x: \mathcal{S} \rightarrow \mathbb{R}$ that specifies how much agent 2 must transfer to agent 1 in each state. Therefore the utility obtained by agent 1 under contract $x$ in state $s$ is given by $V_{1}\left[d_{1}(s)+x(s)\right]$, while the utility to agent 2 under contract $x$ is given by $V_{2}\left[d_{2}(s)-x(s)\right]$. For ease of notation, for the remainder of the paper we set $V_{1}\left[d_{1}(s)+x(s)\right]=U_{1}[x(s), s]$ and $V_{2}\left[d_{2}(s)-x(s)\right]=U_{2}[x(s), s]$.

The maximization problem that yields the first best co-insurance contract between the two agents is now easy to state. For simplicity again we just take it to be the case that agent 1 makes a take-it-or-leave-it offer to agent 2 . We denote by $\bar{U}_{i}$ the reservation level of expected utility of agent $i=1,2$. Since in the absence of a contract the resources are divided according to the default $\left(d_{1}(s), d_{2}(s)\right)$, the reservation levels are set as $\bar{U}_{i}=\sum_{s \in \mathcal{S}} p(s) V_{i}\left[d_{i}(s)\right]$ for $i=1,2$.

The first best contract is then simply the solution to ${ }^{3}$

$$
\begin{aligned}
\max _{x(\cdot)} & \sum_{s \in \mathcal{S}} p(s) U_{1}[x(s), s] \\
\text { s.t. } & \sum_{s \in \mathcal{S}} p(s) U_{2}[x(s), s] \geq \bar{U}_{2} \\
& x(s) \in\left[-d_{1}(s), d_{2}(s)\right] \forall s \in \mathcal{S}
\end{aligned}
$$

Throughout the rest of the paper, the solution to Problem (1) is denoted by $x^{*}$, and we refer to it as the first best contract. ${ }^{4}$ Further, we denote by $U_{1}^{*}$ and $U_{2}^{*}$ the expected utility levels that agents 1 and 2, respectively, achieve. ${ }^{5}$

Sometimes in the analysis that follows we need to refer to the space of 'all possible' contracting problems. Clearly, we could parameterize the set of all possible contracting problems as all the elements of Problem (1) vary: the agents' utility functions, the default $d(\cdot)$, and the probability distribution $p(\cdot)$. This would be unnecessarily cumbersome for what is needed here. In fact for our purposes in this paper, it is enough

\footnotetext{
${ }^{3}$ Notice that under our assumptions the solution to Problem (1) is obviously unique.

${ }^{4}$ Of course the actual values of $x^{*}(s)$ for each $s$ are entirely characterized by the first order conditions associated with Problem (1) and by the first constraint in Problem (1) holding as an equality. This characterization of $x^{*}$ is largely immaterial for the analysis that follows since we will always work taking the first best contract as given.

${ }^{5}$ Of course it is immediate that $U_{2}^{*}=\bar{U}_{2}$.
} 
to parameterize the set of possible contracting problems by keeping the agents' utility functions fixed, and just letting the default $d(\cdot)$ and the probability distribution $p(\cdot)$ vary. This is what we will do throughout the rest of the paper.

\section{A Definition of Contractual Incompleteness}

As we mentioned above, we would like a definition of contractual incompleteness that splits the set of all possible contracts into two disjoint exhaustive sets: the contracts that are complete and those that are incomplete. We would also like the definition of incompleteness to capture the idea that incomplete contracts are those contracts that show evidence that the contracting parties were constrained in their ability to distinguish between states when the contract was drawn up.

Notice that agents may be constrained in their ability to distinguish between states in a way that makes the constraint not binding. In other words they may be unable to distinguish between states that even unconstrained agents would want to treat equally in the contract they write. In this case there will be no evidence of the informational constraints in the contract that the agents draw up when we compare it with the first best. Hence, the case of non-binding informational constraints will be indistinguishable from that of agents who are not constrained in their ability to distinguish between different states of nature. In the case of non-binding informational constraints, our definition below will end up classifying the contract as a complete one.

On the other hand some possible contracts may distinguish 'too much' between different states of nature. It is possible to envision contracts that are more variable (as a function of the state of nature) than the first best. These contracts will clearly yield the contracting parties a level of expected utility that is below the first best level. However, whatever the reason that may compel the contracting parties to draw up such contracts, they will clearly present no evidence that the agents were constrained in their ability to distinguish between states when the contract was drawn up. In these cases too our definition of incompleteness below will classify the contract as a complete one.

We start our analysis with an analogy that in our view is suggestive since it invokes the familiar categories of a complete and of an incomplete set of securities in 
the text-book sense of the word.

Notice that a contract $x: \mathcal{S} \rightarrow \mathbb{R}$ can always be thought of as a portfolio of state-contingent securities. Suppose now that a complete set of securities is available to the agents in the sense that all Arrow securities can be used. In other words the contracting parties are allowed to choose the sharing rule as any linear combination of the following set of elementary securities. For every $i \in \mathbb{N}$ let

$$
x_{i}(s)=\left\{\begin{array}{cc}
1 & \text { if } s=i \\
0 & \text { otherwise }
\end{array}\right.
$$

It is then clear that the sharing rule chosen would be exactly the first best sharing rule $x^{*}$ solving Problem (1).

Imagine now that an incomplete set of securities is available to the agents because some states cannot be distinguished from others. The structure of securities available that we have in mind is of the following type. Let $\mathcal{P}$ be a partition of the state space $\mathcal{S}$ which is strictly coarser than the finest possible one. Let also $\mathcal{I}_{i}(i=1, \ldots, n, \ldots)$ be a typical element of $\mathcal{P}{ }^{6}$ Consider now the problem of finding a sharing rule as any linear combination of the following set of securities. For every $i=1, \ldots, n, \ldots$ let

$$
x_{i}^{\mathcal{P}}(s)=\left\{\begin{array}{cc}
1 & \text { if } s \in \mathcal{I}_{i} \\
0 & \text { otherwise }
\end{array}\right.
$$

Of course it may still be the case that the contracting parties are able to choose a portfolio of such restricted set of securities which is exactly the first best sharing rule. However, in general, this will not be the case. If the problem of finding a portfolio of a restricted set of securities as above yields a solution which is different from the first best, we call the resulting contract incomplete.

Lastly, we notice that the set of contracts that one would call incomplete according to the above intuitive reasoning may be too small. The problem is the following. Consider the solution to the appropriate version of Problem (1) when the parties are restricted to choose among portfolios of a restricted set of securities as above and

\footnotetext{
${ }^{6}$ Since $\mathcal{S}$ is countably infinite, the number of elements of a partition $\mathcal{P}$ of $\mathcal{S}$ can be at most countably infinite.
} 
assume that this yields an incomplete contract. Consider now a new contract that induces exactly the same partition of the state space as the above, but which takes the 'wrong' values so that it cannot possibly be an optimal portfolio for any restricted set of securities. One would still call such a contract incomplete. Such contract is 'wrong' given the parties probability beliefs and utility functions as well as being incomplete. In other words, we would like the definition of incomplete contract to refer only to the partition of the state space which a contract induces and not to the values which the contract takes.

Some additional notation is needed at this point. Let $\Pi$, with typical element $\mathcal{P}$, be the set of all possible partitions of the state space $\mathcal{S}$. Given a partition $\mathcal{P}$ and any $s \in \mathcal{S}$, let $\mathcal{I}(\mathcal{P}, s) \subseteq \mathcal{S}$ be the element (the 'cell') of the partition $\mathcal{P}$ that contains state $s$. The finest possible partition of $\mathcal{S}$ is denoted by $\mathcal{P}^{*}$, so that $\mathcal{I}\left(\mathcal{P}^{*}, s\right)=\{s\}$ for every $s \in \mathcal{S}$.

Given any possible contract $x(\cdot)$, let $P(x) \in \Pi$ be the partition of the state space $\mathcal{S}$ that the contract $x$ induces. In other words, $P(x)$ is defined by setting, for every $s \in \mathcal{S}$,

$$
\mathcal{I}[P(x), s]=\left\{s^{\prime} \in \mathcal{S} \text { such that } x\left(s^{\prime}\right)=x(s)\right\}
$$

When comparing two partitions of the state space $\mathcal{P}$ and $\mathcal{P}^{\prime}$, we will use the notation $\mathcal{P} \succcurlyeq \mathcal{P}^{\prime}$ to denote the fact that $\mathcal{P}$ is equal to or coarser than $\mathcal{P}^{\prime}$, which is of course equivalent to saying that $\mathcal{P}^{\prime}$ is equal to or finer than $\mathcal{P}$.

Lastly, we need to set up some notation to identify what contract would be drawnup by two agents who are constrained by the fact that they cannot distinguish between any two states that are in the same cell of a given partition $\mathcal{P}$. To this end, we start by setting up the appropriate version of Problem (1) when the contracting parties are constrained by the partition $\mathcal{P}$. Given any $\mathcal{P} \in \Pi$, consider

$$
\begin{array}{ll}
\max _{x(\cdot)} & \sum_{s \in \mathcal{S}} p(s) U_{1}[x[\mathcal{I}(\mathcal{P}, s)], s] \\
\text { s.t. } & \sum_{s \in \mathcal{S}} p(s) U_{2}[x[\mathcal{I}(\mathcal{P}, s)], s] \geq \bar{U}_{2} \\
& x[\mathcal{I}(\mathcal{P}, s)] \in\left[-d_{1}(s), d_{2}(s)\right] \forall s \in \mathcal{S}
\end{array}
$$


where of course $x(\cdot)$ is now a function taking the elements of $\mathcal{P}$ into $\mathbb{R}$. Let $x(\mathcal{P})$ be the sharing rule that solves Problem (5). ${ }^{7}$

Clearly, for any $\mathcal{P} \in \Pi, x(\mathcal{P})$ is the best co-insurance contract that can be drawnup by two agents who are constrained by $\mathcal{P}$ in the sense that they are unable to distinguish between any two states that are in the same element of $\mathcal{P}$.

We are now ready to ask formally the question of whether, given a contract $x$, there is any evidence that the contracting parties who drew-up $x$ were constrained by a partition $\mathcal{P}$ in a binding way. For any possible contract $x$, let

$$
\Pi(x)=\{\mathcal{P} \in \Pi \text { such that } P[x(\mathcal{P})] \succcurlyeq P(x)\}
$$

In other words, $\Pi(x)$ is the set of partitions of the state space with the following property. If we constrain the agents' ability to distinguish among states by a partition $\mathcal{P}$ that is in $\Pi(x)$, and then we look at the resulting contract and at the partition that it induces (namely $P[x(\mathcal{P})]$ ), we obtain a partition of the state space that is no finer than the one induced by the original contract $x$. In effect, $\Pi(x)$ is the set of partitions which, when used as a constraint, could possibly induce the agents to draw up a contract that distinguishes among states just as finely as $x$, or less finely than $x$ does.

Clearly, if we find that for a given $x$ we have that $\mathcal{P}^{*} \in \Pi(x)$ we have no evidence that the agents who drew up contract $x$ were constrained in any way in their ability to distinguish among states. Indeed in this case two contracting parties who could distinguish among any two states of nature, would have drawn up a contract that partitions the state space as finely as $x$, or less finely than $x$ does. So, $x$ may perhaps be 'too sensitive' to the realization of the state of nature, but there is no evidence that the agents were unable to distinguish between any two states of nature.

Consider on the other hand a contract $x$ for which we find that $\mathcal{P}^{*} \notin \Pi(x)$. In this case, by definition, we know that two agents who could distinguish between any two states could not possibly have drawn-up the contract $x$, nor any contract that distinguishes less finely between states than $x$ does. Therefore we can conclude

\footnotetext{
${ }^{7}$ It is clear that under our assumptions on the agents' utility functions the solution to Problem (5) is unique.
} 
that $x$ does indeed contain evidence of the fact that the agents who drew it up were somehow constrained in their ability to distinguish between states. If the agents had been granted the ability to distinguish between any two states, they would have used it to draw up a contract that partitions the state space in a different way.

To sum up, we state explicitly the definition of complete and incomplete contracts that we have in effect already given. Broadly speaking, a contract is incomplete if and only if it does not incorporate some information about the state of nature that it would have been optimal for the contracting parties to include.

Definition 1. Incomplete Contracts: A contract is complete if $\mathcal{P}^{*} \in \Pi(x)$, and is incomplete if $\mathcal{P}^{*} \notin \Pi(x)$.

Definition 1 can be cumbersome to use since it involves constructing an entire set of partitions $\Pi(x)$, and then checking whether $\mathcal{P}^{*}$ belongs to the set. Fortunately, there is an equivalent way to state Definition 1 that is easier to use.

REMARK 1. Incompleteness and First Best: A contract $x$ is incomplete according to Definition 1 if and only if

$$
P\left(x^{*}\right) \nsucc P(x)
$$

In other words, a contract is incomplete if and only if the partition of the state space that it induces is not the same as, or finer than, the partition induced by the first best contract $x^{*}$.

We conclude this Section with an observation. Roughly speaking, if a contract is incomplete, then it must be sub-optimal in the obvious sense. However, a contract that is complete need not be optimal in any way. Some complete contracts are suboptimal in a straightforward way. They partition the state space perhaps even more finely than the first best, but the values of the sharing rule that they embody are not the optimal ones.

REMARK 2. Incompleteness and Sub-Optimality: Any contract that is incomplete according to Definition 1, and which yields agent 2 an expected utility level that 
is no less than $\bar{U}_{2}$, must yield agent 1 a level of expected utility that is strictly less than the first best level $U_{1}^{*}$.

There exist some contracts that are complete according to Definition 1, which yield agent 2 an expected utility level equal to $\bar{U}_{2}$, and at the same time give agent 1 a level of expected utility that is strictly less than the first best level $U_{1}^{*}$.

\section{Computable Contracts}

The bounded rationality of the agents in our world is embodied in a constraint that the contracts they write must be algorithmic in nature. We use what in the mathematical literature is widely accepted to be the widest possible notion of an algorithmic or effectively computable. ${ }^{8}$ A function is computable if it can be computed by an abstract computing device known as a Turing machine. Any function that can be computed in a finite number of steps by any imaginable finite device can in fact be computed by a Turing machine. This claim is known in the mathematical literature as Church's thesis. ${ }^{9}$ In this sense, when we assume that the contracts that our agents stipulate are computable, we are simply assuming that they must be objects of a finite level of complexity, however complexity is defined.

A Turing machine is identified by its program. In turn a program is a finite string of symbols drawn from a finite alphabet that obeys some syntactical rules that we do not specify in detail here. ${ }^{10}$ The machine's input also consists of a finite string of symbols drawn from a finite alphabet, and is placed on a 'tape' at the start of the computation. The machine then manipulates the contents of the tape according to its program. Whatever is on the tape when the machine halts (if it ever halts) is taken to be the result of the computation.

What matters for our purposes here is to notice that, since a Turing machine is identified by a finite string of symbols, the set of all possible Turing machines can be put in a one-to-one (computable) correspondence with the set of natural numbers $\mathbb{N}$. Throughout the rest of the paper each Turing machine is identified with its 'code' in $\mathbb{N}$ which is thus obtained. It is also important to notice at this stage that

\footnotetext{
${ }^{8}$ Throughout the paper we use the terms algorithmic, effectively computable, computable and Turing computable in an interchangeable way.

${ }^{9}$ See for instance Cutland (1980), or Hopcroft and Ullman (1979).

${ }^{10}$ See the references in footnote 9 or for a brief exposition Anderlini (1989).
} 
the computation of a Turing machine on a given input is not guaranteed to halt: the computation can 'loop' and carry on ad infinitum. ${ }^{11}$ In this case the machine's output is undefined.

The output of a Turing machine on a given input (provided the computation halts of course) is itself a finite string of symbols drawn from a finite alphabet. However, recall that the contracting problem that we described in Section 2 above requires the contract $x$ to specify a real number (a transfer from agent 2 to agent 1 ) for each state of nature $s$.

Since the output of a Turing machine is finite, we take it to be the 'code' of a rational number (the two integers that define the given rational). ${ }^{12}$ Throughout the rest of the paper the set of rational numbers is denoted by $\mathbb{Q}$ while the set of nonnegative rational numbers is denoted by $\mathbb{Q}_{+}$. The output of a Turing machine (when it is defined) $x \in \mathbb{N}$ on an input $y \in \mathbb{N}$ will be denoted by $\varphi_{x}(y)$.

We are now ready to define the set of computable contracts for the contracting problem described in Section 2 above.

Definition 2. Computable Contracts: A computable contract is a Turing machine $x \in \mathbb{N}$ such that for every $s \in \mathcal{S}$ the output $\varphi_{x}(s)$ is defined. Moreover it must be the case that $\varphi_{x}(s) \in \mathbb{Q} \cap\left[-d_{1}(s), d_{2}(s)\right]$ for every $s \in \mathcal{S}$. Throughout the rest of the paper, the set of computable contracts is denoted by $\mathcal{C} \subset \mathbb{N}$

\section{The Approximation Result}

A simple counting argument is sufficient to show that if we restrict our agents to choose a computable contract from the set $\mathcal{C}$, in some cases the contract they choose will necessarily be different from the first best contract $x^{*}$. There are uncountably many possible first best contracts, while $\mathcal{C}$ is a countable set. Moreover, there are uncountably many ways in which the first best can partition the state space, while $\mathcal{C}$, again, is a countable set. It is possible that the first best contract partitions the state space in a way that cannot possibly be matched by any computable contract

\footnotetext{
${ }^{11}$ This is a case that we will rule out by assumption in our analysis below.

${ }^{12}$ Clearly there are many ways to code the rational numbers into $\mathbb{N}$. The details of this operation are completely inessential for the analysis that follows. We simply assume that this operation is carried out in a given, fixed, way throughout the rest of the paper.
} 
$x \in \mathcal{C}$. This is sufficient to show that in some cases, any computable contract will be different from the first best.

REMARK 3. Computability and the First Best: There exist some contracting problems (i.e. there exist some possible defaults $d(\cdot))$ such that any possible computable contract $x \in \mathcal{C}$ induces a partition of the state space that is different from the one induced by the first best.

Remark 3 does not assert that all possible computable contracts will be incomplete for the class of contracting problems that it identifies. This is in fact not the case for the following reason. Recall that according to Remark 1 a contract that partitions the state space more finely than the first best is complete according to Definition 1. Since it is easy to construct computable contracts that partition the state space in the finest possible way (they induce the partition $\mathcal{P}^{*}$ ), it is then clear that just the fact that the partition induced by the first best cannot be matched by any computable contract is not sufficient to guarantee that all computable contracts will be incomplete.

It is evident that any contract that partitions the state space more finely than the first best is dominated in a straightforward way. Both agents are risk averse, and therefore 'excess partitioning' results in lower expected utilities. It turns out that if we eliminate those computable contracts that partition the state space more finely than needed, Remark 3 can be strengthened to obtain contracts that are actually incomplete.

Definition 3. Dominated Computable Contracts: A computable contract $x \in \mathcal{C}$ is dominated within $\mathcal{C}$ if and only if there exists another computable contract $x^{\prime} \in \mathcal{C}$ that yields a higher level of expected utility to both agents.

Our next remark asserts that for some contracting problems all computable contracts that are not dominated within $\mathcal{C}$ are in fact incomplete.

REMARK 4. Incompleteness and Computability: There exist some contracting problems (i.e. there exist some defaults $d(\cdot))$ such that any possible computable contract $x \in \mathcal{C}$ that is not dominated within $\mathcal{C}$ is incomplete according to Definition 1. 
Notice that Remark 2 is silent as to whether the incompleteness that it identifies really matters in terms of the agents' expected utility levels. The natural question to ask at this point is the following. Is it the case that when choosing a contract from $\mathcal{C}$ our agent's expected utilities will be necessarily bounded away (below of course) from what they can achieve using the first best contract? Or is it the case that, whatever the contracting problem, there are computable contracts that yield the agents levels of expected utility that are in fact arbitrarily close to their first best levels? The rest of this section is devoted to showing that the answer to the latter question is yes. ${ }^{13}$

Proposition 1. The Approximation Result: Let any contracting problem and any $\varepsilon>0$ be given. Then there exists a computable contract $x \in \mathcal{C}$ that yields to each agent a level of expected utility within $\varepsilon$ of their first best levels $U_{1}^{*}$ and $U_{2}^{*}$.

The intuition behind Proposition 1 is not hard to outline. First of all notice that the fact that the output of a computable contract is restricted to be a rational number rather than a real clearly should not prevent a computable contract from approximating the first best. The rational numbers are dense in the reals so that the values of the first best can be approximated pointwise by a function taking only rational values.

The second observation that clarifies the logic behind Proposition 1 is the following. Any countably additive probability distribution $p(\cdot)$ over the natural numbers must have a tail in the sense that for any $\xi>0$ there must exist an $n$ such that $\sum_{s=n}^{\infty} p(s)<\xi$. It follows that, since utilities are assumed to be bounded below, failure to approximate the values of the first best for all $s>n$ entails a maximum expected utility loss that is proportional to $\xi$. Since $\xi$ can be made arbitrarily small, it now follows that it is sufficient to approximate the values of the first best over a finite subset of the possible states of nature. To close the argument it is then enough to notice that any function from $\mathbb{N}$ into $\mathbb{Q}$ (appropriately coded into $\mathbb{N}$ of course) that is constant everywhere except over a finite subset of $\mathbb{N}$ is in fact computable. Therefore, picking an appropriate cut-off point $n$, and approximating the values of the first best up to $s=n$ while assigning an arbitrary value to the sharing rule for

\footnotetext{
${ }^{13}$ In Anderlini and Felli (1994) we showed that this claim is true in a model with a continuum of states of nature. In that paper too, we refer to this fact as the Approximation Result.
} 
$s>n$ we can approximate the first best contract by means of a computable contract as closely as desired. ${ }^{14}$

\section{Complexity Costs}

\subsection{Preliminaries}

The Approximation Result of Section 5 tells us that any first best can be approximated by a computable contract. ${ }^{15}$ Restricting attention to contracts that are computable as in Definition 2 provides a natural model of what feasible contracts can achieve. On the other hand, the Approximation Result asserts that, in terms of the parties' expected utilities, the restriction to computable contracts is not binding. Thus, the problem of obtaining genuine contractual incompleteness in this set up is not a trivial one. Our next step is to model explicitly the complexity costs associated with a contract. This, in turn, will be sufficient to generate genuine contractual incompleteness.

Recall that a computable contract is a contract that specifies a sharing rule that can be computed in a finite number of steps by an abstract computing device (a Turing machine). While the number of steps in each computation yielding the value of the sharing rule in each state of nature must be finite, there is no bound on the number of steps that the computation may involve. In a sense, the Approximation Result applies in a 'limit case' of bounded rationality. Computations must yield an answer in finite time, but no extra resources are needed to carry out more and more complex computations.

The natural next question to ask is whether the Approximation Result applies to a world in which actual complexity costs are explicitly taken into account. The framework of computable contracts provides a natural setting to address this question since it already embodies the view that a contract is an object that maps the

\footnotetext{
${ }^{14} \mathrm{We}$ will come back to this construction and to the Approximation Result in Sections 7 and 8 below.

${ }^{15}$ Some of the material in this section of the paper parallels the results reported in Anderlini and Felli (1999). Notice however that in Anderlini and Felli (1999) we work with a finite state space throughout, while the results stated here apply to both the case of a finite state space and to the case explicitly treated here of $\mathcal{S}$ that coincides with $\mathbb{N}$. Moreover, the assumptions on complexity costs that we use in the paper are substantially weaker than those used in Anderlini and Felli (1999). In particular, the complexity costs in Anderlini and Felli (1999) are assumed to take a minimum value (normalized to 1) for any computation. This is not the case here. As a result the arguments behind our results in this Section are a great deal more intricate than in Anderlini and Felli (1999).
} 
possible realizations of the state of nature into the values of a sharing rule via a well defined computation. All that is needed is to attach a complexity measure to the computations that are carried out.

The obvious problem with modeling complexity costs is that the results that one can obtain are often specific to the particular form that complexity costs are assumed to take. In the case of a contracting situation this is a particularly unpleasant feature of many possible modeling options. The complexity costs that a contract involves may not only be specific to the contracting parties reasoning abilities, but will inevitably be specific to the legal system in which the contract is embedded. ${ }^{16}$

To deal with the problem we have just outlined we pursue the following modeling strategy. Instead of assuming a specific form for the complexity costs that a contract entails, we simply specify a set of properties that the complexity costs must satisfy. We then look for results that characterize the impact of complexity costs for any complexity measure in the class that we have so defined.

\subsection{The Contracting Problem}

In order to introduce explicitly complexity costs into Problem (1), we need to modify the original contracting problem in two ways. First of all, we need to introduce a complexity cost function, and secondly we need to incorporate these costs into the 'accounting identities' of the problem.

Throughout this section we assume that the default allocation $d(\cdot)$ is a computable function of the state of nature $s$. This is completely unnecessary to our results below. We choose to proceed in this way because it streamlines the analysis and it avoids setting-up a considerable amount of extra notation.

Definition 4. Computable Default: A computable default allocation is a pair of Turing machines $d=\left(d_{1}, d_{2}\right) \in \mathbb{N}^{2}$ such that for every $s \in \mathcal{S}$ both outputs $\varphi_{d_{i}}(s)$ are defined and belong to $\mathbb{Q}_{+}$.

\footnotetext{
${ }^{16}$ In Anderlini and Felli (1999) we quote the example of the procedure to change a person's name. In the United kingdom this is an extremely simple procedure (Deed Poll). In Italy, however, it is and extremely complex one (often denied anyway) that involves a sentence of the Court of Appeal.
} 
We can now proceed to define the complexity costs of a contract in a given state. ${ }^{17}$ The set of computable contracts $\mathcal{C}$ is a natural one over which to define complexity costs. Given a computable contract $x$ and a state $s$, we can interpret the associated complexity cost as a cost associated with the computation that Turing machine $x$ carries out on input $s$.

Given our discussion above, it now seems natural to define complexity costs as being given by a computable function that maps the computable contract $x$ and the state $s$ into a non-negative rational number $c \in \mathbb{Q}_{+} \cdot{ }^{18}$

Definition 5. Complexity Costs: A set of complexity costs for a contracting problem is a pair of Turing machines $c=\left(c_{1}, c_{2}\right) \in \mathbb{N}^{2}$. Each Turing machine takes as an input the pair $(x, s)$ and the output $\varphi_{c_{i}}(x, s) \in \mathbb{Q}_{+}$is assumed to be defined whenever $x \in \mathcal{C}$ and $s \in \mathcal{S}$.

The non-negative rational number $\varphi_{c_{i}}(x, s)$ is interpreted as the complexity cost accruing to agent $i$ from the computable contract $x$ in state $s$.

We will come back to the properties that a set of complexity costs function is assumed to satisfy in Subsection 6.3 below. For the time being, we simply restate the basic contracting problem when complexity costs are taken into account. Recall that we have defined the functions $U_{i}(\cdot, \cdot)$ using $V_{1}\left[d_{1}(s)+x(s)\right]=U_{1}[x(s), s]$ and $V_{2}\left[d_{2}(s)-x(s)\right]=U_{2}[x(s), s]$. Therefore we have that $V_{1}\left[\varphi_{d_{1}}(s)-\varphi_{c_{1}}(x, s)+\varphi_{x}(s)\right]=$ $U_{1}\left[\varphi_{x}(s)-\varphi_{c_{1}}(x, s), s\right]$ and symmetrically $V_{2}\left[\varphi_{d_{2}}(s)-\varphi_{c_{2}}(x, s)-\varphi_{x}(s)\right]=U_{2}\left[\varphi_{x}(s)+\right.$ $\left.\varphi_{c_{2}}(x, s), s\right]$. The relevant version of Problem (1), yielding the optimal computable

\footnotetext{
${ }^{17}$ Notice that while we are defining the complexity costs of a contract on a state-by-state basis, our formalism is clearly compatible with some contracts having some 'fixed costs' that apply to all states. Such fixed costs would simply have to be incorporated in the complexity costs of a given contract in every state $s$.

${ }^{18}$ Of course, one could imagine the complexity cost of a contract in a given state to depend on the value of the default pair $\left(\varphi_{d_{1}}(s), \varphi_{d_{2}}(s)\right)$ as well. We do not do this purely for the sake of simplicity. All our results in this section remain true when this dependency is allowed.
} 
contract given a set of complexity costs $c$ reads as follows.

$$
\begin{aligned}
\max _{x \in \mathcal{C}} & \sum_{s \in \mathcal{S}} p(s) U_{1}\left[\varphi_{x}(s)-\varphi_{c_{1}}(x, s), s\right] \\
\text { s.t. } & \sum_{s \in \mathcal{S}} p(s) U_{2}\left[\varphi_{x}(s)+\varphi_{c_{2}}(x, s), s\right] \geq \bar{U}_{2} \\
& \varphi_{d_{1}}(s)-\varphi_{c_{1}}(x, s)+\varphi_{x}(s) \geq 0 \quad \forall s \in \mathcal{S} \\
& \varphi_{d_{2}}(s)-\varphi_{c_{2}}(x, s)-\varphi_{x}(s) \geq 0 \quad \forall s \in \mathcal{S}
\end{aligned}
$$

The last two constraints in Problem (8) state that neither agent should receive less than zero in any state of nature, after the default, the complexity costs and the contract are taken into account. Recall that we have set $r(s)=\varphi_{d_{1}}(s)+\varphi_{d_{2}}(s)$ for every $s$, and notice that adding up the last two constraints in Problem (8) we obtain immediately that

$$
r(s)=\varphi_{d_{1}}(s)+\varphi_{d_{2}}(s) \geq \varphi_{c_{1}}(x, s)+\varphi_{c_{2}}(x, s) \quad \forall s \in \mathcal{S}
$$

so that we know that the total of complexity costs cannot exceed the total amount of resources available in any state of nature.

Finally, notice that, at this point, we do not know whether there are any computable contracts that are even feasible in Problem (8). This will be the case after we have made suitable assumptions on the form of the set of complexity $\operatorname{costs} c$.

If a solution to Problem (8) does indeed exist it will be denoted by $x_{c}^{*}$ and we will refer to it as the optimal computable contract given complexity $\operatorname{costs} c$. The first best contract for Problem (8) is denoted by $x^{*}$ as before. Of course $x^{*}$ can be thought of as the solution (not necessarily a computable contract) to Problem (8) when complexity costs are set identically equal to zero in every state.

\subsection{Axiomatic Complexity Costs}

As we mentioned in our brief discussion above, we do not wish to specify the complexity cost function $c$ in full. We take an 'axiomatic' approach in this respect. We simply state some properties that the set of complexity costs $c$ must satisfy, and then characterize the impact of complexity costs on the contracting problem for any $c$ in the class that these properties define. 
The first property that we require $c$ to satisfy is simply that the 'null' contract carries no complexity costs at all. A contract that does not prescribe any transfers between agents 1 and 2 has a cost of zero in every state of nature.

Assumption 1. Zero Costs for Null Contract: Let $x_{0} \in \mathcal{C}$ be a given Turing machine that computes the null contract so that $\varphi_{x_{0}}(s)=0$ for every $s$ in $\mathcal{S} .{ }^{19}$

The set of complexity costs function $c \in \mathbb{N}^{2}$ is such that $\varphi_{c_{i}}\left(x_{o}, s\right)=0$ for every $s \in \mathcal{S}$ and for every $i=1,2$.

The second property that we require $c$ to satisfy is best explained by recourse to an analogy. Imagine that the complexity costs, for each state $s$, measure the 'number of steps' required by $x$ to compute the output $\varphi_{x}(s)$. Initially, the input $s$ is placed on the tape of Turing machine $x$. Then the machine starts to manipulate the contents of the tape. The content of the tape after the machine halts is then taken to be the result of the computation $\varphi_{x}(s)$. During each step only a finite set of manipulations are possible. Suppose now that we were to require that the computation should take a maximum of, say, $y$ steps. Then clearly there should be a finite number of possible configurations of the tape by the time the computation has halted. In other words, for every possible input and for every possible bound, the output of the computation $\varphi_{x}(s)$ is restricted to be in some finite set. This will in fact be the case for every complexity cost function that allows only 'finite increments' in the computation for each increase in the complexity cost.

The formal counterpart of the property we have just described intuitively is our next assumption on the complexity costs in Problem (8).

Assumption 2. Computational Increments: The set of complexity costs $c \in \mathbb{N}^{2}$ has the following property. For every $i=1,2$, for every $y \in \mathbb{Q}_{+}$and for every $s \in \mathcal{S}$ there exists a non-empty finite set $\Theta_{i}(y, s) \subset \mathbb{Q}$ such that

$$
\varphi_{c_{i}}(x, s) \leq y \quad \Rightarrow \quad \varphi_{x}(s) \in \Theta_{i}(y, s)
$$

\footnotetext{
${ }^{19}$ Notice that there are a countable infinity of Turing machines in $\mathcal{C}$ that compute the null contract as above. We simply require that our assumption holds for at least one such Turing machine.
} 
We stated above that in general a solution, or even a feasible computable contract in Problem (8) is not guaranteed to exist. ${ }^{20}$ Our next remark summarizes the situation when Assumptions 1 and 2 hold.

Remark 5. Feasibility and Supremum: Under Assumptions 1 and 2 a solution to Problem (8) may or may not exist.

On the other hand, a feasible computable contract $x \in \mathcal{C}$ for Problem (8) is guaranteed to exist.

Therefore, even when a solution does not exist, the supremum over $x \in \mathcal{C}$ of the maximand in Problem (8) is well defined. This supremum (regardless of whether it coincides with the maximum) will be denoted by $\tilde{U}_{1}$.

Our next concern is to study the impact of complexity costs satisfying Assumptions 1 and 2 on the underlying co-insurance problem. As we know from Remark 4 above, for some co-insurance problems any computable contract is necessarily incomplete. This of course implies that for some contracting problems the first best contract is not computable. To disentangle the effect of the non-computability of the first best from the effect of the complexity costs, we characterize the impact of complexity costs restricting attention to contracting problems that yield a first best contract that is in fact computable. For the sake of completeness, our next step is to define formally this class of co-insurance problems.

Definition 6. Computable First Best: A contracting problem is said to yield a computable first best if and only if the solution $x^{*}$ to Problem (1) is computable in the sense that there exist $x \in \mathcal{C}$ such that $x^{*}(s)=\varphi_{x}(s)$ for every $s$ in $\mathcal{S}$.

We are now ready to state our next result. It amounts to saying that the Approximation Result no longer holds when we take into account any set of complexity costs that satisfies Assumptions 1 and 2.

\footnotetext{
${ }^{20}$ Notice that it is easy to show that when the state space $\mathcal{S}$ is finite a solution to Problem (8) always exists. We omit the details of the argument for the sake of brevity and since our focus here is on a countably infinite state space.
} 
Proposition 2. Sub-Optimality with Complexity Costs: Let any set of complexity costs satisfying Assumptions 1 and 2 be given. Then there exist some contracting problems (i.e. there exist some computable default $d \in \mathbb{N}^{2}$ and some probability distribution $p(\cdot))$ which yield a computable first best and such that the supremum of the expected utility achievable by agent $1, \tilde{U}_{1}$ is strictly below the first-best level $U_{1}^{*}$.

Of course, Proposition 2 is silent as to whether the optimal computable contract (if it exists) will be incomplete or not. The sub-optimality of the contract that the agents draw up could simply be due to their inability, given the complexity costs, to stipulate the 'right values' for the sharing rule $x$. On the other hand, it could be that the complexity costs prevent them from partitioning the states of nature in the correct way, in which case the resulting contract will be incomplete. Which of these two possibilities induces the sub-optimality of the contract that the agents draw up, in general, is determined by the specific form of the complexity costs $c$.

Proposition 2 can be strengthened. In fact we can show that given any complexity costs satisfying Assumptions 1 and 2 we can find some contracting problems for which the optimal computable contract given the complexity costs exists and is incomplete in the strong sense that it is the null contract that prescribes no transfers between agents 1 and 2. The class of contracting problems for which this occurs is smaller than the class of contracting problems identified by Proposition 2 for two reasons. First of all, we know from Remark 4 that not all contracts that are sub-optimal are in fact incomplete according to Definition 1. Secondly, Proposition 3 below asserts that the optimal computable contract with complexity costs is the null contract. In general, for a given contracting problem the set of contracts that are incomplete according to Definition 1 contains contracts that are different from the null contract as well as the null one. ${ }^{21}$

In essence the class of contracting problems identified by Proposition 3 below is the class of co-insurance problems for which the first best contract is 'close' to the null contract, relative to the 'scale' of the complexity costs. If this is the case the parties, faced with complexity costs $c$ will write no contract at all. Our next proposition is the main endogenous incompleteness result of this paper.

\footnotetext{
${ }^{21}$ Of course in the special case of a contracting problem for which the null contract is the first best the null contract is in fact complete.
} 
Proposition 3. Incompleteness with Complexity Costs: Let any set of complexity costs $c$ satisfying Assumptions 1 and 2 be given. Then there exist some contracting problems (i.e. there exist some computable default $d \in \mathbb{N}^{2}$ and some probability distribution $p(\cdot))$ with the following properties.

The contracting problem yields a computable first best, which is different from the null contract. An optimal computable contract given complexity costs c exists and it coincides with the null contract that prescribes no transfer between agents 1 and 2 in every state of nature.

It follows from Definition 1 that for these contracting problems the optimal computable contract given complexity costs $c$ is incomplete.

\section{Some Related Results}

This section is devoted to a discussion of two papers that are related to the present one: Battigalli and Maggi (2000) and Krasa and Williams (1999).

Battigalli and Maggi (2000) are directly concerned with the complexity costs associated with a contract. They start by specifying an explicit model of the language that the parties can use in drawing up a contract. To every phrase in this language they associate a level of complexity costs. This, in turn, defines a level of complexity costs associated with each possible contract. They then proceed to characterize the optimal contract selected by the parties and the type of contractual incompleteness that it may display. In particular they distinguish between those situations in which a contract exhibits 'excessive rigidity' and those in which a contract displays 'excessive discretion'.

Battigalli and Maggi (2000) work with a model in which each state is described by a finite string of $0 \mathrm{~s}$ and $1 \mathrm{~s}$. Each of these digits is interpreted as the truth or falseness of one of the finitely many 'elementary statements' available in the parties' language. The possible 'actions' that a contract might prescribe are defined in a similar way. An action is a finite string of $0 \mathrm{~s}$ and $1 \mathrm{~s}$, with the $1 \mathrm{~s}$ corresponding to the finitely many possible 'elementary activities' to be carried out.

A contract is now viewed as a sentence (a 'well formed formula') in the parties language that is assembled from its elementary statements using logical connectives. Each elementary sentence that is used carries a cost that is given and equal for all 
elementary statements. The logical connectives, on the other hand, do not carry a cost.

Once the complexity cost of any contract is specified in this way, it becomes possible to characterize in a simple environment the features of contracts that are chosen optimally (taking the complexity costs into account of course). The optimal contract given complexity costs is then compared with the first best, and the differences between the two are characterized.

Battigalli and Maggi (2000) focus on two characteristics of optimal contracts that are induced by the complexity costs: 'rigidity' and 'discretion.' Roughly speaking, rigidity occurs whenever a contract partitions the state space in a way that is not finer (or equal) than the partition induced by the first best contract (this corresponds to the definition of an incomplete contract proposed in Definition 1 above). Discretion instead occurs whenever the description of the actions prescribed by the contract is coarser than what the first best contract would say. The party whose performance is disciplined by the contract is left discretion on some (or all) of the elementary actions associated with states in a given cell.

Battigalli and Maggi (2000) show that the specification of the complexity costs they use implies that contingent clauses that apply if and only if a given state of nature occurs are the most expensive ones, rigid clauses that apply to all states of nature are less expensive while the actions that are left to each party's discretion are the least expensive of all (they are not regulated at all). The result is that in the simplest formulation of their model, the optimal contract is such that the set of feasible actions can be partition in three groups: the most important actions, regulated by contingent clauses, the less important actions, regulated by rigid clauses and finally the least important actions, left to the discretion of the party to whom the contract applies.

We conclude our brief review of Battigalli and Maggi (2000) with two observations. The first is that a major difference between the work of Battigalli and Maggi (2000) and our analysis here is that while they use a particular specification of the complexity costs, in Section 6 we use an axiomatic approach that encompasses a large class of possible complexity measures. Clearly the choice of one complexity measure affords Battigalli and Maggi a more detailed characterization of the impact that complexity 
costs have on optimal contracts. On the other hand, being specific about the exact form of the complexity costs has a drawback. The results that one derives are only as appealing as the specification of the complexity costs that is used. As we discussed at some length above it seems clear that a complete specification of complexity costs that properly fit a wide variety of contractual situations may be too much to ask.

Our second observation concerning Battigalli and Maggi (2000) is related to the first one. In order to specify completely the form of the complexity costs, Battigalli and Maggi need to specify and take as given the set of elementary statements that the language allows. This of course entails that the set of elementary sentences cannot 'evolve' in a way that allows one 'new' elementary sentences to emerge that are equivalent to, say, two of the original elementary sentences in the language. This process of 're-coding', given the form of complexity costs that Battigalli and Maggi assume, would dramatically change the cost of a given contract. By contrast, in Section 6 we considered a broad class of complexity measures. This allows us to leave unspecified what can be carried out in a single 'step'. Since our results hold for any complexity measure in this broad class, they hold for whatever 're-coding' may have evolved in the parties' language.

A recent paper by Krasa and Williams (1999) is concerned with the a version of the Approximation Result that we described in Section 5 above.

It should be noted that the Approximation Result described in Section 5 applies to a model with a countable state space while in Krasa and Williams (1999) the cardinality of the state space is that of the continuum, so that the Approximation Result they are concerned with is like the one presented in Anderlini and Felli (1994).

As in Anderlini and Felli (1994), in Krasa and Williams (1999) a state is described by an infinite sequence of $0 \mathrm{~s}$ and $1 \mathrm{~s}$, each digit representing the presence or the absence of a particular 'feature' of the state. Critically, in Anderlini and Felli (1994) the underlying state space is taken to be the unit interval $[0,1]$. Therefore, whenever a real number $s \in[0,1]$ has more than one binary representation, an arbitrarily predefined one is chosen. ${ }^{22}$ By contrast, Krasa and Williams (1999) take the state space

\footnotetext{
${ }^{22}$ For instance the two binary numbers $0.1000 \ldots$ and $0.0111 \ldots$ both correspond to the number 1/2. In Anderlini and Felli (1994) the first of these two binary representation of $1 / 2$ is chosen by assumption.
} 
to be the set $\{0,1\}^{\mathbb{N}}$. In this way they allow 'duplicate' representations of a countable infinity of real numbers in the interval $[0,1]$ to be included in the state space.

Krasa and Williams (1999) call a contract incomplete if and only if it conditions its outcome on a finite number of the digits that describe a state of nature. They call a contract recordable (in a given contracting problem) if the utilities that it yields to the contracting parties can be approximated by a sequence of incomplete contracts. In their set-up, they go on to show that a sufficient condition for the optimal contract to be recordable is that the contracting problem exhibits what they call asymptotic decreasing importance. A contracting problem exhibits asymptotic decreasing importance if the impact on utility of the digits of the state of nature decreases to zero as we move further to the right along the sequence of 0 s and 1s. In other words, whenever a contracting problem exhibits asymptotic decreasing importance, a version of the Approximation Result holds.

Krasa and Williams (1999) go on to show that in some contracting problems in which asymptotic decreasing importance does not hold the Approximation Result fails so that, in their terminology, the optimal contract is not recordable. In essence failures of asymptotic decreasing importance can be viewed as discontinuities of the parties utilities in the outcome that the contract prescribes. Intuitively, when this is the case, it may well be the case that approximating the optimal contract by a sequence of incomplete ones fails to approximate the optimal contract in utility terms. The role of discontinuities of this kind, in a standard principal-agent model, had also been recognized in Anderlini and Felli (1998). In this paper too, if the principal's preferences are discontinuous it may be the case that any sequence of 'written' (incomplete in the terminology of Krasa and Williams (1999)) contracts may fail to approximate the utilities yielded by the optimal one. While the focus of Anderlini and Felli (1998) is to characterize the effects of these discontinuities in a principal-agent model, Krasa and Williams (1999) focus on the condition of asymptotic decreasing importance which ensures that such discontinuities do not arise and hence that the Approximation Result holds.

Krasa and Williams (1999) then proceed to consider a situation in which the parties to a contractual problem are asymmetrically informed. Each agent knows only his own type. Each agent's type is an infinite sequence of 0s and 1s. In this 
setting Krasa and Williams (1999) focus on incomplete contracts (defined as before) that are truthful in the sense that each party is asked to reveal his own type up to a certain point in the sequence of 0 and 1 that describes it. It is at this point that the 'duplication' of states that we described above has a bite in their model. Roughly speaking, suppose that two states have the same impact on utility if they correspond to the same real number in $[0,1]$. Then, in the terminology of Krasa and Williams (1999), each digit is reversible in the sense that its effect on utilities can be undone by an infinite sequence of digits that follow it. ${ }^{23}$ If this is the case, the only incomplete contracts that induce truth-telling do not depend on the parties' declarations. Intuitively this is because only a finite number of digits can be reported by each party. Now consider the last of the reported digits. Because of reversibility both a 0 and a 1 are compatible with the truth (in utility terms). Hence the contract must treat equally a report of 0 and 1 as the last digit. Since the outcome of the contract must be independent of the last reported digit, we can now apply the same reasoning to the penultimate digit and, by induction, the outcome of the contract cannot depend on any of the digits reported by the parties. Krasa and Williams (1999) interpret this result as saying that, in the case of asymmetric information, highly incomplete contracts may arise even when asymptotic decreasing importance holds.

\section{The Structure of the State Space and the Approximation Result}

We conclude our discussion of related results with a description of the analysis carried out in Al-Najjar, Anderlini, and Felli (2000).

The main aim of this paper is to investigate a model in which the relevant version of the Approximation Result described in Section 5 fails, not because of a discontinuity in the parties' preferences (as in Anderlini and Felli (1998) and Krasa and Williams (1999)) but because of the intrinsic complexity of the contracting problem.

When the parties utility functions are continuous in 'money' the Approximation Result is very pervasive. In a model with a continuum of states, if we can compute the parties' expected utilities, then the contract must prescribe money transfers between

\footnotetext{
${ }^{23}$ For instance, the state $0.1000 \ldots$ has the same effect on utilities as the state $0.0111 \ldots$ Hence the impact on utilities of 0 in the first position after the decimal point can be undone by an infinite sequence of $1 \mathrm{~s}$ starting in the second position after the decimal point.
} 
the agents that are a (Borel) integrable function of the state of nature. It then follows that any contract that yields well defined payoffs to the contracting parties must be approximable by a sequence of 'step functions'. Since every step function is computable ${ }^{24}$ it then follows that any contract that yields the parties well defined expected utilities (including the first best) can be approximated, in payoff terms, by a sequence of computable contracts. Roughly speaking, in this context, integrability yields the Approximation Result in a direct way.

In a model with a countable state space, whenever utilities are continuous in money, the Approximation Result holds for the reasons we described at some length in Section 5 above. In short, if the probability measure we put on the state space is countably additive, we can select a finite subset of the state space which captures all relevant features of the contracting problem, up to any desired degree of precision.

The setting of Al-Najjar, Anderlini, and Felli (2000) is that of states that are described by an infinite sequence of $0 \mathrm{~s}$ and $1 \mathrm{~s}$. For the reasons described above, the analysis in Al-Najjar, Anderlini, and Felli (2000) is moved away from a world in which there is a continuum of possible states of nature, to a setting in which there is a countable infinity of states. To ensure that the Approximation Result does not hold in the same way as described in Section 5, Al-Najjar, Anderlini, and Felli (2000) place an 'atomless' (finitely additive) measure on the state space.

The main result reported in this paper can now be described as follows. It is possible that in the model we have outlined, the environment exhibits a degree of 'fine variability' that cannot be captured by any contract that conditions its prescriptions on a finite set of the component features of each state. In other words, the environment that the parties are faced with could be so 'complex' in its variability, that any computable contract must leave out a significant proportion of the variability of the first best.

Roughly speaking the main result of Al-Najjar, Anderlini, and Felli (2000) is driven by the fact that the variability of the environment can be made to depend on the 'tail' of digits that describes each state of nature. This has a non-negligible impact on what a computable contract can achieve, not because of discontinuities in

\footnotetext{
${ }^{24}$ See Anderlini and Felli (1994) for a precise definition of what constitutes a computable function in a model with a continuum of states.
} 
the parties' preferences, but because the probability measure on the state space is in fact atomless. This in turn makes it necessary to capture the fine variability of the environment in order to achieve the first best. As a result, computable contracts cannot even approximate the parties' first best expected utilities.

Al-Najjar, Anderlini, and Felli (2000) goes on to provide a tight characterization of which part of the variability of the environment can possibly be captured by a finite (computable) contract. Very intuitively, each contracting environment in the model we have outlined has a 'closest match' in a mirror image model with a continuum of states. Finite contracts can capture (or approximate in some cases) the variability that the continuum mirror image world embodies on sets of positive measure, but not any extra fine variability that is displayed by the discrete world. The translation between the discrete and the continuum worlds that we have just described is in fact sufficient to provide a full characterization of the nature of the optimal finite contracts in the original model with a countable state space.

\section{APPENDIX}

Proof of Remark 1: Assume that a contract $x$ is complete according to (7) so that $P\left(x^{*}\right) \succcurlyeq P(x)$. Notice that clearly we have that $P\left(x^{*}\right)=P\left[x\left(\mathcal{P}^{*}\right)\right]$. Therefore, if $P\left(x^{*}\right) \succcurlyeq P(x)$ it must be that case that $\mathcal{P}^{*} \in \Pi(x)$.

Assume next that a contract $x$ is complete according to Definition 1. Then, by assumption, $P\left[x\left(\mathcal{P}^{*}\right)\right] \succcurlyeq P(x)$. But since $P\left(x^{*}\right)=P\left[x\left(\mathcal{P}^{*}\right)\right]$, this implies directly that $P\left(x^{*}\right) \succcurlyeq P(x)$.

Proof of Remark 2: The first claim is a direct consequence of the fact that, under our assumptions, the first best contract is unique. Indeed, consider any contract $x$ that is incomplete according to Definition 1 and that yields agent 2 a level of expected utility greater or equal to $\bar{U}_{2}$. Using Remark 1 we immediately have that $x(s) \neq x^{*}(s)$ for some $s \in \mathcal{S}$. Therefore, since $x^{*}$ is the unique solution to Problem (1), and $x$ is feasible in Problem (1) it is immediate that $\sum_{s \in \mathcal{S}} p(s) U_{1}[x(s), s]$ $<U_{1}^{*}$.

The proof of the second claim is as follows. Consider the first best contract $x^{*}$. Notice that using the Inada conditions on $V_{1}$ and $V_{2}$ we must have that $d_{2}(s)-x^{*}(s)>0$ and $d_{1}(s)+x^{*}(s)>$ 0 for every $s \in \mathcal{S}$.

Now consider a new contract $\tilde{x}$ obtained from $x^{*}$ as follows. Set $\tilde{x}(s)=x^{*}(s)$ for every $s \in$ $\mathcal{S}$, except for two, arbitrarily fixed, states, $s^{\prime}$ and $s^{\prime \prime} \neq s^{\prime}$. Now choose two (small) positive real numbers, $\epsilon$ and $\xi$, and set $\tilde{x}\left(s^{\prime}\right)=x^{*}\left(s^{\prime}\right)+\epsilon$, and $\tilde{x}\left(s^{\prime \prime}\right)=x^{*}\left(s^{\prime \prime}\right)-\xi$. Moreover, ensure that $\epsilon$ and $\xi$ have the following properties. 
First of all $\epsilon<d_{2}\left(s^{\prime}\right)-x^{*}\left(s^{\prime}\right)$ and $\xi<d_{1}\left(s^{\prime \prime}\right)+x *\left(s^{\prime \prime}\right)$. Secondly $\sum_{s \in \mathcal{S}} p(s) U_{2}[\tilde{x}(s), s]=$ $\sum_{s \in \mathcal{S}} p(s) U_{2}\left[x^{*}(s), s\right]=U_{2}^{*}$. Thirdly $\tilde{x}\left(s^{\prime}\right) \neq \tilde{x}\left(s^{\prime \prime}\right) \neq x^{*}(s)$ for every $s \in \mathcal{S}$.

Notice that $\epsilon$ and $\xi$ can always be chosen as required by continuity of $V_{2}$. Moreover, it is clear that the contract $\tilde{x}$ has the properties stated in the claim. By construction it is feasible in Problem (1) and it yields agent 2 an expected utility level equal to $\bar{U}_{2}=U_{2}^{*}$. Finally, by construction again, $\tilde{x}$ is such that $P\left(x^{*}\right) \succcurlyeq P(\tilde{x})$, so that, using Remark $1, \tilde{x}$ is complete according to Definition 1.

Proof of Remark 3: Notice that the set of all possible partitions of the state space $\Pi$ has the cardinality of the continuum. On the other hand $\mathcal{C}$ is a countable set. Therefore the set of partitions induced by all possible computable contracts is also a countable set. Therefore, $\Pi$ must contain some partitions that are not induced by any possible computable contract $x \in \mathcal{C}$.

Proof of Remark 4: Let the probability distribution $p(\cdot)$ be given. Fix also a total amount of resources $\bar{r}$ constant across states so that $r(s)=d_{1}(s)+d_{2}(s)=\bar{r}$ for every $s \in \mathcal{S}$. Consider the class of contracting problems obtained as the default $d$ varies subject to the constant resource constraint above.

Using the first order conditions of Problem (1) it is now immediate that, whatever the default $d$, the first best contract must satisfy

$$
d_{1}\left(s^{\prime}\right)+x^{*}\left(s^{\prime}\right)=d_{1}\left(s^{\prime \prime}\right)+x^{*}\left(s^{\prime \prime}\right) \quad \text { and } \quad d_{2}\left(s^{\prime}\right)-x^{*}\left(s^{\prime}\right)=d_{2}\left(s^{\prime \prime}\right)-x^{*}\left(s^{\prime \prime}\right) \quad \forall s^{\prime}, s^{\prime \prime} \in \mathcal{S}
$$

Our first step is to show that for any problem in this class, any computable contract that induces a partition that is finer than $P\left(x^{*}\right)$ is dominated within $\mathcal{C}$.

Let a computable contract $x \in \mathcal{C}$ with $P\left(x^{*}\right) \succ P(x)$ be given. Choose $s^{\prime}$ and $s^{\prime \prime}$ in $\mathcal{S}$ so that $x^{*}\left(s^{\prime}\right)=x^{*}\left(s^{\prime \prime}\right)$ and $x\left(s^{\prime}\right) \neq x\left(s^{\prime \prime}\right)$. This must be possible since, by assumption, $P\left(x^{*}\right) \succ P(x)$.

Now consider a new contract $\tilde{x}$ obtained from $x$ as follows. Set $\tilde{x}(s)=x(s)$ for every $s \in \mathcal{S}$ except for $s^{\prime}$ and $s^{\prime \prime}$. Now let $z \in \mathbb{Q}$ be a rational number that is arbitrarily close to

$$
\frac{p\left(s^{\prime}\right) x\left(s^{\prime}\right)+p\left(s^{\prime \prime}\right) x\left(s^{\prime \prime}\right)}{p\left(s^{\prime}\right)+p\left(s^{\prime \prime}\right)}
$$

and set $\tilde{x}\left(s^{\prime}\right)=\tilde{x}\left(s^{\prime \prime}\right)=z$. Clearly, using (A.2) and by concavity of $V_{1}$ and $V_{2}$, the contract $\tilde{x}$ yields higher expected utility to both agents than the contract $x$. Moreover, since $x \in \mathcal{C}$, we can be sure that $\tilde{x}$ is also a computable contract in $\mathcal{C}$. This is because $\tilde{x}$ is obtained from $x$ by changing only a finite set of values of $x$. Hence, by Church's thesis (see footnote 9 ) if $x$ is computable, so is $\tilde{x}$.

Hence we have concluded the proof of the fact that, for any contracting problem within the class considered here, if a computable contract $x$ has the property that $P\left(x^{*}\right) \succ P(x)$, then $x$ is dominated within $\mathcal{C}$. 
Observe next that using (A.1), keeping $\bar{r}$ fixed and varying $d_{1}(\cdot)$ and $d_{2}(\cdot)$ it is evident that we can ensure that the first best partitions the state space in any arbitrary way. In other words, for every $\mathcal{P} \in \Pi$, we can find a default $d$ such that $P\left(x^{*}\right)=\mathcal{P}$. Using the same argument as in the proof of Remark 3 we can now set the default $d$ in such a way that for every $x \in \mathcal{C}$ we have that $P(x) \neq$ $P\left(x^{*}\right)$.

We know from our first step that any computable contract that is not dominated within $\mathcal{C}$ cannot induce a partition of $\mathcal{S}$ that is finer than $P\left(x^{*}\right)$. Therefore, using Remark 1 any computable contract $x$ that is complete and is not dominated within $\mathcal{C}$ must have $P(x)=P\left(x^{*}\right)$. This is clearly a contradiction and hence concludes the proof of our claim.

Proof of Proposition 1: We take it as given that any contract $x: \mathcal{S} \rightarrow \mathbb{Q} \cap\left[-d_{1}(s), d_{2}(s)\right]$ that is constant except for a finite subset of states $\overline{\mathcal{S}} \subset \mathcal{S}$ is in fact computable. ${ }^{25}$

For every $s \in \mathcal{S}$ let $z(s)$ be a rational number arbitrarily close to $x^{*}(s)$. For every $n$ consider the contract $x_{n}$ defined as $x_{n}(s)=z(s)$ for every $s \leq n$, and $x_{n}(s)=0$ for every $s>n$. By our first assertion above, $x$ is a computable contract for every $n$ finite.

Since $\sum_{s \leq n} p(s)$ approaches 1 as $n$ approaches $\infty$, and since $z(s)$ is chosen to be arbitrarily close to $x^{*}(s)$ for every $s$, clearly as $n$ grows without bound the parties' expected utilities from the sequence of computable contracts $x_{n}$ approach their first best levels $U_{1}^{*}$ and $U_{2}^{*}$ as required.

Proof of Remark 5: The set of computable contracts that are feasible in Problem (8) is clearly not empty because of Assumption 1. The rest of the claim follows immediately from this observations and the details are therefore omitted.

Lemma A.1: Consider the following class of co-insurance problems parameterized by a single real, $\lambda$ to be defined below.

Let $r(s)$ be a constant $\bar{r}>0$ and set the default $d$ as follows. Fix arbitrarily an $n>0$ and for every $s \leq n$, let $d_{1}(s)=0$ and $d_{2}(s)=\bar{r}$. For every $s>n$, let $d_{1}(s)=\bar{r}$ and $d_{2}(s)=0$.

Let $p=\sum_{s \leq n} p(s)$. Consider next any $\lambda \in[0, \bar{r}]$ and set

$$
p=\frac{V_{2}(\lambda)-V_{2}(0)}{V_{2}(\bar{r})-V_{2}(0)}
$$

Then the first best contract $x_{\lambda}^{*}$ has the following form.

$$
x_{\lambda}^{*}(s)=d_{2}(s)-\lambda \quad \forall s \in \mathcal{S}
$$

\footnotetext{
${ }^{25}$ This is an elementary result in recursive function theory. See the references in footnote 9.
} 
Proof: By completely standard arguments the first best must have the property that

$$
d_{2}\left(s^{\prime}\right)-x_{\lambda}^{*}\left(s^{\prime}\right)=d_{2}\left(s^{\prime \prime}\right)-x_{\lambda}^{*}\left(s^{\prime \prime}\right) \quad \forall s^{\prime}, s^{\prime \prime} \in \mathcal{S}
$$

Notice next that, using (A.3), the reservation expected utility level of agent 2 reads as follows.

$$
\bar{U}_{2}=p V_{2}(\bar{r})+(1-p) V_{2}(0)=V_{2}(\lambda)
$$

Since the first best must guarantee a level of expected utility of precisely $\bar{U}_{2}$ to agent 2 , combining (A.5) and (A.6) yields (A.4), as required.

Lemma A.2: Consider the parametric class of co-insurance problems of Lemma A.1. Assume that $\bar{r}$ is a positive rational number, and let the parameter $\lambda$ range over the rationals $[0, \bar{r}] \cap \mathbb{Q}$.

This new parametric class of co-insurance problems has the following properties.

(i) It contains a countable infinity of elements.

(ii) Every co-insurance problem in the class has a computable default.

(iii) Every co-insurance problem in the class yields a computable first best.

(iv) For every arbitrarily fixed $z \in(0, \bar{r}) \cap \mathbb{Q}$ there exists a co-insurance problem in the class such that $x_{\lambda}^{*}(1)=\bar{r}-z$.

Proof: Property (i) follows from the fact that $[0, \bar{r}] \cap \mathbb{Q}$ contains a countable infinity of elements. Property (ii) follows directly from the definition of the defaults (which are fixed as $\lambda$ varies) in Lemma A.1. Property (iii) follows directly from (A.4). Property (iv) also follows immediately from (A.4) by letting $z=\lambda$.

Lemma A.3: Let any set of complexity costs satisfying Assumptions 1 and 2 be given.

Then, for every $s \in \mathcal{S}$ there exists a finite set $\Theta(s) \subset \mathbb{Q}$ such that, for every computable contract that is feasible in Problem (8) we have that $\varphi_{x}(s) \in \Theta(s)$.

Proof: If a computable contract is feasible in Problem (8) it must satisfy (9). The statement then follows immediately from Assumption 2. 
Proof of Proposition 2: Let a set of complexity costs $c$ satisfying Assumptions 1 and 2 be given.

Recall that the complexity costs $c$ yield a sequence of finite sets $\Theta(s)$ as in Lemma A.3.

Consider next the class of co-insurance problems of Lemma A.2. Recall that every problem in this class yields a computable first best, as required.

Suppose now that the statement of the proposition were false. Then for every problem in the class, for every $s$ and for every $\epsilon>0$, we should be able to find a computable contract $x$ that is feasible in Problem (8) and such that $\left\|\varphi_{x}(1)-x_{\lambda}^{*}(1)\right\|<\epsilon$. However, given that $\varphi_{x}(1) \in \Theta(1)$, this is impossible given property (iv) of Lemma A.2.

Lemma A.4: Consider the following class of co-insurance problems parameterized by a single real, $z \in(1,1 / 2)$ to be defined below.

Let $r(s)$ be a constant $\bar{r}>0$ and set the default $d$ as follows. Fix arbitrarily an $n^{\prime}>0$ and $n^{\prime \prime}$ $>n^{\prime}$. For every $s \leq n^{\prime}$, let $d_{1, z}(s)=(1-z) \bar{r}$ and $d_{2, z}(s)=z \bar{r}$. For every $n^{\prime}<s \leq n^{\prime \prime}$, let $d_{1, z}(s)=$ $z \bar{r}$ and $d_{2, z}(s)=(1-z) \bar{r}$, and finally for every $s>n^{\prime \prime}$ let $d_{1, z}(s)=d_{2, z}(s)=(1 / 2) \bar{r}$.

Assume that the probability distribution $p(\cdot)$ is such that

$$
\sum_{n^{\prime}<s \leq n^{\prime \prime}} p(s)=\frac{1}{4}
$$

Let $p=\sum_{s \leq n} p(s)$ and set

$$
p=\frac{1}{4} \frac{V_{2}((1 / 2) \bar{r})-V_{2}((1-z) \bar{r})}{V_{2}(z \bar{r})-V_{2}((1-z) \bar{r})}
$$

Then the first best contract $x_{z}^{*}$ has the following form.

$$
x_{z}^{*}(s)=d_{2, z}(s)-\frac{1}{2} \bar{r} \quad \forall s \in \mathcal{S}
$$

Proof: By completely standard arguments the first best must have the property that

$$
d_{2, z}\left(s^{\prime}\right)-x_{\lambda}^{*}\left(s^{\prime}\right)=d_{2, z}\left(s^{\prime \prime}\right)-x_{\lambda}^{*}\left(s^{\prime \prime}\right) \quad \forall s^{\prime}, s^{\prime \prime} \in \mathcal{S}
$$

Notice next that, using (A.8), the reservation expected utility level of agent 2 reads as follows.

$$
\bar{U}_{2}=p V_{2}(z \bar{r})+(1 / 4) V_{2}((1-z) \bar{r})+((3 / 4)-p) V_{2}((1 / 2) \bar{r})=V_{2}((1 / 2) \bar{r})
$$

Since the first best must guarantee a level of expected utility of precisely $\bar{U}_{2}$ to agent 2 , combining (A.10) and (A.11) yields (A.9), as required. 
Lemma A.5: Consider the parametric class of co-insurance problems of Lemma A.4. Assume that $\bar{r}$ is a positive rational number, and let the parameter $z$ range over the rational numbers $(1,1 / 2) \cap \mathbb{Q}$. This new parametric class of co-insurance problems has the following properties.

(i) Every co-insurance problem in the class has a computable default.

(ii) Every co-insurance problem in the class yields a computable first best.

(iii) For every $\epsilon>0$ there exists a co-insurance problem in the class such that $x_{z}^{*}(s)<\epsilon$ for every $s$ in $\mathcal{S}$.

(iv) For every problem in the class $x_{z}^{*}(s)=0$ for every $s>n^{\prime \prime}$

Proof: Property (i) follows directly from the definition of the defaults in Lemma A.4. Property (ii) follows directly from (A.9). Properties (iii) and (iv) also follow immediately from (A.9).

Proof of Proposition 3: Let a set of complexity costs $c$ satisfying Assumptions 1 and 2 be given.

We will now proceed to show that the statement of the proposition is true for any problem in the class identified in Lemma A.5 when $z$ is sufficiently close to $1 / 2$.

Notice first of all that by Lemma A.5 we know that any such problem has a computable default and yields a computable first best, as required. Notice further that since $z>1 / 2$, using (A.9) we also know that any such problem yields a first best that is different from the null contract, as required.

Using (A.9) we can see that, as $z$ approaches $1 / 2$, the null contract $x_{0}$ yields the parties levels of expected utilities that approach their respective first best levels $\bar{U}_{1}$ and $\bar{U}_{2}$ respectively. This is the case because the first best approaches the null contract point-wise.

The rest of the argument proceeds by contradiction. Suppose that the statement of the proposition is false. Then for every $z \in(1,1 / 2)$ there must exist a computable contract $x_{z}$ that is different from $x_{0}$, that is feasible in Problem (8) and which yields agent 1 a level of expected utility that is above the level of expected utility given by the null contract $x_{0}$. Using the fact that complexity costs are always non-negative, and the fact that, by Assumption 1, the contract $x_{0}$ carries a complexity cost of zero in every state for both contracting parties we can now conclude the following. If the statement of the proposition is false, for every $z \in(1,1 / 2)$ we must be able to find a computable contract $x_{z}$ that is feasible in Problem (8) and such that

$$
\begin{aligned}
\mathcal{V}_{z}^{*}=\sum_{s \in \mathcal{S}} p(s) V_{1}\left(d_{1, z}+x_{z}^{*}(s)\right) & > \\
\mathcal{V}_{z}\left(x_{z}\right) & =\sum_{s \in \mathcal{S}} p(s) V_{1}\left(d_{1, z}(s)+\varphi_{x_{z}}(s)\right)> \\
\mathcal{V}_{z}\left(x_{0}\right) & =\sum_{s \in \mathcal{S}} p(s) V_{1}\left(d_{1, z}(s)+\varphi_{x_{0}}(s)\right)
\end{aligned}
$$


As we noted already, as $z$ approaches $1 / 2$, we know that $\mathcal{V}_{z}\left(x_{0}\right)$ approaches $\mathcal{V}_{z}^{*}$. Using (A.12) this implies that, as $z$ approaches $1 / 2$ we must have that $\mathcal{V}_{z}\left(x_{z}\right)$ also approaches $\mathcal{V}_{z}^{*}$. By continuity of $V_{1}$ and $V_{2}$ and by uniqueness of the first best, this implies that the contract $x_{z}$ must approach the first best contract point wise, as $z$ approaches $1 / 2$.

Since, by assumption, $x_{z}$ is feasible in Problem (8), using Lemma A.3 it is immediate that

$$
\forall s \in \mathcal{S} \quad \exists z_{s}>\frac{1}{2} \text { such that } z \leq z_{s} \Rightarrow \varphi_{x_{z}}(s)=0
$$

Using (A.13) we can now define

$$
\bar{z}=\min _{s \leq s^{\prime \prime}} z_{s}
$$

Therefore we know that

$$
z \leq \bar{z} \Rightarrow \varphi_{x_{z}}(s)=0 \quad \forall s \leq s^{\prime \prime}
$$

Notice next that it is straightforward to verify that the null contract $x_{0}$, solves Problem (8) with the additional constraint that $\varphi_{x}(s)=0$ for every $s \leq s^{\prime \prime}$. Therefore, from (A.15), since $x_{z}$ is feasible in Problem (8), we can now conclude that

$$
z \leq \bar{z} \Rightarrow \mathcal{V}_{z}\left(x_{0}\right) \geq \mathcal{V}_{z}\left(x_{z}\right)
$$

Since (A.16) obviously contradicts (A.12) the proposition is now proved.

\section{REFERENCES}

Aghion, P., And J. Tirole (1997): "Formal and Real Authority in Organizations," Journal of Political Economy, 105, 1-29.

Al-NajJar, N., L. Anderlini, and L. Felli (2000): "Incomplete Contracts in a Complex World," mimeo.

Anderlini, L. (1989): "Some Notes on Church's Thesis and the Theory of Games," Theory and Decision, 29, 19-52.

Anderlini, L., And L. Felli (1994): "Incomplete Written Contracts: Undescribable Sates of Nature," Quarterly Journal of Economics, 109, 1085-1124. (1998): "Describability and Agency Problems," European Economic Review, $42,35-59$. 
(1999): "Incomplete Contracts and Complexity Costs," Theory and Decision, $46,23-50$.

Battigalli, P., And G. Maggi (2000): "Imperfect Contracting," mimeo.

Blum, M. (1967): "A Machine-Independent Theory of the Complexity of Recursive Functions," Journal of the Association of Computing Machinery, 14, 322-336.

Cutland, N. J. (1980): Computability: An Introduction to Recursive Function Theory. Cambridge: Cambridge University Press.

Grossman, S. J., And O. D. Hart (1986): "The Costs and Benefits of Ownership: A Theory of Vertical and Lateral Integration," Journal of Political Economy, 94, $691-719$.

Hart, O. D., And J. Moore (1988): "Incomplete Contracts and Renegotiation," Econometrica, 56, 755-85.

(1990): "Property Rights and the Nature of the Firm," Journal of Political Economy, 98, 1119-58.

(1999): "Foundations of Incomplete Contracts," Review of Economic Studies, $66,115-138$.

Hopcroft, J. E., AND J. Ullman (1979): Introduction to Automata Theory, Languages and Computation. London: Addison-Wesley.

Krasa, S., AND S. R. Williams (1999): "Incompleteness as a Constraint in Contract Design," mimeo.

Maskin, E., And J. Tirole (1999): "Unforseen Contingencies and Incomplete Contracts," Review of Economic Studies, 66, 83-114.

Rajan, R., And L. Zingales (1998): "Power in a Theory of the Firm," Quarterly Journal of Economics, 113, 387-432.

REICHE, S. (1999): "Foundations of Incomplete Contracts: Asymmetric Information and Renegotiation," mimeo. 
Segal, I. (1999): "Complexity and Renegotiation: A Foundation for Incomplete Contracts," Review of Economic Studies, 66, 57-82.

Tirole, J. (1999): "Incomplete Contracts: Where Do We Stand?," Econometrica, 67, $741-781$. 